\section{Getting to the essence of Staph}

\section{By Chris Cain, Staff Writer}

Two recent studies from The University of Chicago have identified vaccine candidates to prevent Staphylococcus aureus infection. ${ }^{1,2}$ The researchers think their strategy of targeting essential surface-localized virulence factors could generate better results than current vaccination approaches, which have yet to show success in humans.

The hypothesis will now need to be put to the test by subjecting the new vaccines to clinical safety and efficacy trials.

Both studies were led by Olaf Schneewind, professor and chair of the Department of Microbiology, who has been focused on identifying proteins on the $S$. aureus cell surface that are required for virulence. His team has been examining essential virulence factors that modulate the host response to infection, hypothesizing that antibodies against those factors will be good candidates to provide protective immunity.

The goal of the first study was to design a nontoxic version of Staphylococcal protein $\mathrm{A}$, an essential virulence factor that suppresses the immune system by killing $B$ cells and by binding and neutralizing immunoglobulins. The research group mutated each of the domains necessary for the immune system interaction and generated a protein A variant lacking these toxic properties.

In mice, the variant provided significant protection against lethal systemic challenge with a methicillin-resistant strain of S. aureus, as measured by decreased kidney bacterial load and abscess formation, compared with that in mock-immunized control mice.

Mice immunized with the variant also had higher antibody titers against a panel of $27 \mathrm{~S}$. aureus cell surface proteins than control mice, suggesting that vaccination could protect the immune system from the suppressive effects of protein A during infection.

"The science behind protein A vaccination clearly demonstrates it can provide a protective effect," Schneewind told SciBX. "The obvious issue is safety, and this nontoxic variant will hopefully solve that problem."

In a second study, Schneewind's team turned to coagulases, which are proteins secreted by $S$. aureus that activate prothrombin and cause clotting. $S$. aureus secretes two coagulases, and the researchers showed that deletion of either coagulase delayed time to death in mice given the modified bacteria compared with in mice given wild-type bacteria.

Deleting both coagulases from the bacteria significantly delayed death in mice $(p<0.0001)$, demonstrating that coagulation is important for $S$. aureus virulence.

In addition, mice immunized with both purified coagulases and challenged with lethal $S$. aureus infection had significantly delayed time to death compared with mock-immunized control mice.

"The coagulase results are also clear, and safety is not a concern with these proteins," said Schneewind. "The complication is that coagulases have diverged significantly across $S$. aureus isolates. The challenge will be to design an antigen that provides immunity against $S$. aureus strains carrying diverse coagulases."

Findings from the two studies were published in The Journal of Experimental Medicine and PLoS Pathogens.

\section{Staph stampede}

To date only one vaccine candidate has completed a Phase III trial, with disappointing results. In 2005, Nabi Biopharmaceuticals' StaphVax missed the primary endpoint of decreasing the incidence of $S$. aureus infection in a Phase III trial in 3,600 end-stage renal disease (ESRD) patients. StaphVax was composed of two capsular polysaccharide antigens, a vaccine design that has been successful in commonly used pneumococcal and meningococcal vaccines.

Nabi, which wasn't available for comment, last year sold its next-generation PentaVax pentavalent $S$. aureus vaccine program to GlaxoSmithKline plc for \$20 million up front and up to \$26 million in milestones in order to focus on NicVax, a nicotine vaccine designed to aid smoking cessation.

Another company that has worked in the area is Inhibitex Inc. The company has decided to focus on antivirals for shingles and HCV and is seeking a partner to develop Aurexis, a mAb against clumping factor $\mathrm{A}$ used in combination with antibiotic treatment, according to Joseph Patti, CSO and SVP of R\&D.

Aurexis completed Phase IIa trials in 2005 in 60 patients with complicated S. aureus bacteremia. Pfizer Inc. has worldwide rights to candidate antigens identified by Inhibitex via a 2001 licensing deal with Wyeth, which Pfizer acquired in 2009.

Merck \& Co. Inc. is conducting the largest clinical trial of an S. aureus vaccine to date. The company's V710 is in the Phase II portion of a Phase II/III study that is immunizing about 8,000 patients undergoing cardiothoracic surgery. The primary endpoint is the prevention of serious S. aureus infections for 90 days following surgery.

V710 is a single-component vaccine that uses iron-regulated hemeiron binding protein IsdB (isdB), a hemoglobin receptor that increases S. aureus survival by scavenging iron from the blood and promoting resistance to neutrophil microbicides. Merck acquired exclusive rights to the antigen in 2004 under a deal with Intercell AG.

"Vaccine efficacy has been demonstrated in animal models. Immunization with V710 improved survival and reduced S. aureus sepsis, as well as deep wound and catheter-associated infections in murine studies. Phase I studies also demonstrated that V710 is well-tolerated and immunogenic," said Dalya Guris, director of clinical research at Merck.

Both of Schneewind's studies were funded by Novartis Vaccines and Diagnostics. The University of Chicago has filed patent applications covering this work.

Cain, C. SciBX 3(39); doi:10.1038/scibx.2010.1168

Published online Oct. 7, 2010 


\section{ANALYSIS}

\section{TARGETS \& MECHANISMS}

\section{REFERENCES}

1. Kim, H.K. et al. J. Exp. Med.; published online Aug. 16, 2010; doi:10.1084/jem.20092514

Contact: Olaf Schneewind, The University of Chicago, Chicago, III. e-mail: oschnee@bsd.uchicago.edu

2. Cheng, A.G. et al. PLoS Pathog.; published online Aug. 5, 2010; doi:10.1371/journal.ppat.1001036

Contact: Olaf Schneewind, The University of Chicago, Chicago, III. e-mail: oschnee@bsd.uchicago.edu
COMPANIES AND INSTITUTIONS MENTIONED

GlaxoSmithKline plc (LSE:GSK; NYSE:GSK), London, U.K. Inhibitex Inc. (NASDAQ:INHX), Alpharetta, Ga.

Intercell AG (VSE:ICLL; OTCQX:INRLY), Vienna, Austria Nabi Biopharmaceuticals (NASDAQ:NABI), Rockville, Md. Merck \& Co. Inc. (NYSE:MRK), Whitehouse Station, N.J.

Pfizer Inc. (NYSE:PFE), New York, N.Y.

The University of Chicago, Chicago, III. 